\title{
Orientações Clínicas para a Abordagem de Doentes Neuromusculares Durante a Pandemia COVID-19
}

\section{Guidelines for Management of Patients with Neuromuscular Disorders During COVID-19 Pandemic}

\author{
Isabel Conceição ${ }^{1,2, *}$, Catarina Falcão de Campos $^{1,2,3}$, Miguel Oliveira Santos ${ }^{1,2,3}$, Mamede de Carvalho ${ }^{1,2,3}$ \\ 1-Serviço de Neurologia, Departamento de Neurociências e Saúde Mental, Hospital de Santa Maria, Centro Hospitalar Universitário \\ Lisboa Norte, Lisboa, Portugal. \\ 2-Instituto de Medicina Molecular, Faculdade de Medicina, Universidade de Lisboa, Lisboa, Portugal. \\ 3-Instituto de Fisiologia, Faculdade de Medicina, Universidade de Lisboa, Lisboa, Portugal.
}

\author{
Informações/Informations: \\ Orientações Clínicas, publicado \\ em Sinapse, Volume 20, \\ Número 2, abril-junho 2020. \\ Versão eletrónica em www. \\ sinapse.pt \\ Guidelines, published in \\ Sinapse, Volume 20, Number \\ 2, april-june 2020. Electronic \\ version in www.sinapse.pt \\ (C) Autor (es) (ou seu (s) \\ empregador (es)) 2020. \\ Reutilização permitida de \\ acordo com CC BY-NC. \\ Nenhuma reutilização \\ comercial. \\ (C) Author(s) (or their \\ employer(s)) 2020. Re-use \\ permitted under CC BY-NC. \\ No commercial re-use.
}

\section{Palavras-chave:}

COVID-19;

Doenças Neuromusculares.

\section{Keywords:}

COVID-19;

Neuromuscular Diseases.

\author{
*Autor Correspondente / \\ Corresponding Author: \\ Isabel Conceição \\ CHULN, \\ Hospital de Santa Maria \\ Avenida Professor Egas Moniz \\ 1649-035 Lisboa, Portugal \\ imsconceicao@gmail.com
}

Recebido / Received: 2020-05-02 Aceite / Accepted: 2020-05-28 Publicado / Published: 2020-07-13

DOI: https://doi.org/10.46531/ sinapse/AR/COVID19/

Conceicaol/2020

\section{Resumo}

A atual pandemia da doença do coronavírus 2019 (COVID-19) levou à necessidade de uma reestruturação dos cuidados de saúde com inevitável impacto na abordagem e tratamento dos doentes neuromusculares. Este grupo de doentes constitui um grupo de maior risco para desenvolver formas graves de COVID-19, quer por características relacionadas com a doença neuromuscular subjacente, quer pelo tratamento imunossupressor, frequentemente administrado neste grupo de patologias, quer ainda pelas co-morbilidades associadas. Assim, a decisão de iniciar, manter ou alterar um tratamento deve pesar o risco para o doente, e para a sociedade, contudo sem negar o seu melhor tratamento possível.

Neste artigo, são apresentadas recomendações, baseada em opiniões de peritos na área, de forma a fornecer orientação para profissionais de saúde no tratamento de doentes neuromusculares.

\footnotetext{
Abstract

The coronavirus-19 disease (COVID-19) pandemic has resulted in reorganization of healthcare settings with potential impact in the delivery of clinical care to patients with neuromuscular disorders. These patients may harbour an increased risk of severe complications related to COVID-19. Several risk factors are identified such as respiratory weakness, cardiac involvement, immunosuppression and presence of comorbidities, among others. Therapeutic decisions must be considered in the best interest of the patients, and the society, with no denial of the best treatment possible.

Clinical recommendations, based on expertise opinion, are presented for clinical care of patients with neuromuscular disorders.
} 


\section{Introdução}

A doença do coronavírus 2019 (COVID-19), atualmente classificada como pandemia pela Organização Mundial da Saúde (OMS), é uma doença sem terapêutica ou vacinação eficaz, de momento.

Com um quadro clínico caracterizado por síndrome respiratória aguda com graus de gravidade variáveis, a sua manifestação mais grave é a síndrome respiratória aguda grave (SARS), com uma mortalidade associada de aproximadamente $3 \%-7 \%$.'

São atualmente reconhecidos os indivíduos com maior risco de desenvolver quadros clínicos mais graves associados a maior mortalidade, em particular, as faixas etárias acima dos 70 anos, doentes com comorbilidades como a diabetes, doença cardíaca, tabagismo e doença respiratória, e doentes imunossuprimidos. ${ }^{2,3}$

À data de hoje, não existem dados sobre risco acrescido de doentes com patologia neuromuscular em pessoas com doenças neuromusculares, com ou sem terapêuticas imunossupressoras.

Doentes com patologia neuromuscular podem estar significativamente em maior risco de desenvolver formas graves de COVID-19, independentemente do diagnóstico subjacente, em particular em doentes com uma ou mais das seguintes características:

I. Relevante fraqueza muscular respiratória (inspiratória ou expiratória), em particular em doentes com capacidade vital inferior a $60 \%$ do predito $(\mathrm{CV}<60 \%)$;

2. Doentes sobre ventilação não-invasiva (NIV), ventilação invasiva, ou com equipamentos auxiliares da tosse

3. Fraqueza muscular bulbar

4. Imunossupressão

5. Atingimento cardíaco

6. Presença de cifoscoliose marcada condicionando alteração da mecânica ventilatória

7. Presença de outras comorbilidades, como doença pulmonar crónica, hipertensão pulmonar, patologia cardíaca, diabetes, neutropenia, linfopenia, doença hepática crónica, gravidez, e idade avançada.

Dentro das doenças neuromusculares, os doentes sob terapêutica imunossupressora têm um risco adicional de apresentar manifestações mais graves de COVID-19.

Este documento, baseado em opiniões de peritos na área pretende fornecer orientação para os profissionais de saúde.

\section{Imunossupressão e doenças neuromusculares em indivíduos sem sintomas de COVID-19}

Não se recomenda que nos doentes com doença ativa sob tratamento, este seja interrompido ou modificado, pois o risco de agravamento/descompensação da sua doença excede o eventual risco acrescido resultante da toma do medicamento. A decisão de alterar ou interromper uma terapêutica imunossupressora deve ser da responsabilidade do médico assistente.

Recomenda-se o distanciamento social com auto-isolamento, assim como o uso de máscara e medidas estritas de higienização (em particular nas saídas obrigatórias do domicilio), para os indivíduos com risco mais elevado decorrente da medicação. ${ }^{4}$

\section{i. Imunoglobulinas e Plasmaferese}

Atualmente, não há evidência que indique risco acrescido para os doentes sob tratamento com imunoglobulina intravenosa ou subcutânea (IVIG, SCIG), assim como plasmaferese.

Relativamente aos doentes sob tratamento crónico com IVIG, a necessidade da sua administração regular, sem interrupção transitória, deve ser considerada pelo médico assistente, tendo em conta a incidência regional de COVID-19 e no risco / benefício da terapêutica para o doente (caso a caso). A eventual conveniência da modificação para tratamento com SCIG deverá ser escrutinada. Nos casos de exacerbação aguda, o julgamento clínico julgará da oportunidade do tratamento tendo em conta os elementos acima.

\section{ii. Corticosteróides}

Admite-se que doses de prednisolona superior a 20 mg diários (ou equivalente) possa constituir um adicional fator de risco para gravidade no caso de COVID-19, em particular quando em associação com outros imunossupressores.

No entanto, reitera-se que nos doentes sob terapêutica com corticosteróides, esta não deve ser suspensa ou modificada, em particular de forma súbita, a menos que especificamente discutido e aprovado pelo médico assistente.

\section{iii. Azatioprina, Micofenelato de Mofetil e Metotrexato}

Doentes medicados com qualquer um destes fármacos (associados ou não aos corticosteróides) devem manter a sua terapêutica habitual, a menos que indicado 
de outro modo pelo médico assistente.

Admite-se que estes medicamentos possam constituir um adicional fator de risco para gravidade no caso de COVID-19. No entanto, em geral, esse risco é superado pelos benefícios da medicação para a doença para o qual está medicado.

\section{Rituximab}

É reconhecido que fármacos depletores celulares, como o rituximab, aumentam moderadamente o risco de infecções virais, estando assim os doentes mais propensos a COVID-19 e suas complicações.

Em muitos doentes, esse risco é superado pelos benefícios do fármaco no tratamento da sua doença, pelo qual deve manter a medicação. No entanto, se considerado seguro para o doente, os tratamentos agendados devem ser julgados quando à oportunidade do adiamento ou da modificação do esquema terapêutico.

Recomenda-se o distanciamento social com auto-isolamento, assim como o uso de máscara e medidas estritas de higienização (em particular nas saídas obrigatórias do domicilio), para os indivíduos com risco mais elevado decorrente da medicação.

2. O que considerar ao iniciar uma terapêutica imunológica em doentes com NM ativa no momento atual?

As recomendações internacionais para o tratamento de uma doença neuromuscular imunomediada devem manter-se de acordo com as melhores práticas clinicas.

Pode ser aconselhável adiar o início das terapêuticas que depletoras celulares (rituximab), até que o pico do surto termine, em cada região. No entanto, o risco de não iniciar a terapêutica de depleção celular em doentes com situações clínicas mais graves pode superar o risco de infeção grave por COVID-19.

\section{Imunossupressão e doenças neuromusculares em indivíduos com o diagnóstico de COVID- 19}

A maioria dos doentes que desenvolvem COVID-I9 têm doença leve e devem continuar com o tratamento indicado para a sua doença.

Em caso de sintomas acentuados, as eventuais alterações terapêuticas resultarão do melhor julgamento clínico no momento.

\section{RECOMENDAÇÕES EM DOENÇAS NEURO- MUSCULARES ESPECÍFICAS}

\section{MIASTENIA GRAVIS (MG)}

A maioria dos doentes com MG está sob terapêutica imunossupressora e/ou imunomoduladora, podendo coexistir fraqueza muscular respiratória, fatores que potenciam o risco de manifestações mais graves de $\mathrm{CO}$ VID-19. Doses reduzidas de corticóides (equivalente a $<20 \mathrm{mg} /$ prednisolon/dia) não estão associadas a risco acrescido de complicações em caso de COVID-19. Não há evidência científica que sugira que terapêuticas sintomáticas, como a piridostigmina, aumentem o risco de infeção ou suas complicações. Relativamente à toma de medicamentos imunossupressores, ver I.iii acima.

Sugerimos que as decisões terapêuticas devam ser individualizadas e tomadas de acordo com o médico assistente. Os doentes com MG devem seguir as diretrizes nacionais emanadas pelas autoridades de saúde.

Doentes de MG sob tratamento médico são aconselhados a não interromper nenhum medicamento, a menos que especificamente discutido e aprovado pelo seu médico.

Nos casos de miastenia generalizada sintomática, recomenda-se o distanciamento social com auto-isolamento, assim como o uso de máscara e medidas estritas de higienização (em particular nas saídas obrigatórias do domicilio). ${ }^{5}$

\section{GLICOGENOSES}

Doentes com doença de Pompe apresentam risco elevado de infeção grave COVID-19 se associado a insuficiência respiratória ( $\mathrm{VC}<60 \%)$; $\mathrm{VNI}$; tosse fraca e cardiomiopatia. Nestes casos, recomenda-se o distanciamento social com auto-isolamento, assim como o uso de máscara e medidas estritas de higienização (em particular nas saídas obrigatórias do domicilio).

A terapêutica de manutenção com alglucosidade, realizada em meio hospitalar implica deslocação a estas unidades, tornando-se incompatível com o distanciamento social requerido, devendo a decisão de adiar o tratamento ser baseada na incidência regional de $\mathrm{CO}$ VID-19 e no risco / benefício da terapêutica para o doente (caso a caso).

\section{AMILOIDOSE HEREDITÁRIA}

Doentes com neuropatia ou cardiomiopatia heredi- 
tária associada a amiloidose TTR (paramiloidose) apresentam um risco elevado para infeção grave relacionada com COVID-19, nas seguintes condições:

I. Estádios mais avançados de doença (estádio III)

2. Sob terapêutica imunossupressora (doentes transplantados)

3. Coexistência de cardiomiopatia com insuficiência cardíaca associada

4. Mau estado nutricional

5. Envolvimento renal com necessidade de hemodiálise.

Aos doentes transplantados/imunossuprimidos aplica-se o descrito acima em I.iii.

Os doentes com envolvimento cardíaco não devem suspender qualquer medicação crónica utilizada para o controle do envolvimento cardíaco.

Doentes sob terapêuticas modificadoras de doença, nomeadamente estabilizadores da TTR (tafamidis) devem manter a sua terapêutica habitual, não sendo reconhecido qualquer risco adicional associado à toma do medicamento.

Doentes sob terapêutica com inotersen (subcutâneo) devem manter a terapêutica, salientando-se a necessidade de manter o controlo analítico quinzenal (hemograma). Sendo este um requisito de segurança requerido para a manutenção da terapêutica, este procedimento deve ser mantido em laboratórios perto da área de residência do doente, ou preferencialmente através de colheitas domiciliárias, e enviado ao médico assistente pelo meio mais conveniente.

Doentes sob terapêutica com patisiran (ev cada 3 semanas), realizada em meio hospitalar implica deslocação a estas unidades, tornando-se incompatível com o distanciamento social requerido, devendo a decisão de adiar o tratamento ser baseada na incidência regional de COVID-19 e no risco / benefício da terapêutica para o doente (caso a caso).

\section{ATROFIA MUSCULAR ESPINHAL}

Doentes com SMA TIPO III apresentam um risco elevado de infeção grave COVID-19 se associado se associado a Insuficiência respiratória (VC $<60 \%)$; VNI; ou tosse fraca.

A terapêutica de manutenção com nusinersen, realizada em meio hospitalar implica deslocação a estas unidades, tornando-se incompatível com o distanciamento social requerido, devendo a decisão de adiar o trata- mento ser baseada na incidência regional de COVID-19 e no risco / benefício da terapêutica para o doente (caso a caso), não sendo reconhecido qualquer risco adicional associado à toma do medicamento. ${ }^{6}$

\section{NEUROPATIAS IMUNOMEDIADAS}

Não se recomenda que nos doentes com doença ativa sob tratamento, a terapêutica seja interrompida ou modificada, pois o risco de agravamento/descompensação da sua doença excede o risco da manutenção do tratamento.

Ao alterar ou interromper uma terapêutica imunossupressora existente que possua um potencial risco para o aumento da atividade e / ou exacerbação da doença de base, devem ser considerados pelo médico assistente os riscos específicos (por exemplo, idade, comorbilidades, localização geográfica (incidência COVID-19) e benefícios. ${ }^{7}$

Nos casos de introdução de terapêutica imunomoduladora, por exemplo em doentes com diagnóstico inaugural, a IGIV deve ser considerada como primeira linha seguida da plasmaferese como terapêutica de segunda linha, quando disponível. A corticoterapia passa a terapêutica de terceira linha, tendo em conta o maior risco de complicações associadas a COVID-19.7

\section{ESCLEROSE LATERAL AMIOTRÓFICA (ELA)}

Nos doentes com ELA e compromisso respiratório (fraqueza dos músculos inspiratórios e/ou expiratórios), em particular naqueles que necessitam de $\mathrm{VNI}$ e de equipamentos de suporte da tosse, qualquer infecção respiratória tem maior risco de complicações médicas, como no caso do COVID-19. Nesta fase deve ser favorecido as consultas por telefone com o médico assistente.

Recomenda-se o distanciamento social com autoisolamento, assim como o uso de máscara cirúrgica e medidas estritas de higienização (em particular nas saídas obrigatórias do domicilio).

É aconselhado a redução ao mínimo do número de cuidadores e evitar visitas. O uso de NIV ou de equipamentos de tosse assistida favorece a aerossolização, pelo que recomenda-se o uso de máscara pelo cuidador para a sua própria proteção. $\mathrm{O}$ apoio destes equipamentos é efectuado por técnicos credenciados que conhecem as medidas adequadas.

Em caso de sintomas de infecção devem ser cumpridas as normas emanadas pela autoridade de Saúde. Deve ser ponderada o uso de filtro nas máscaras do 
equipamento de NIV em caso de o doente ter sinais de infecção respiratória.

\section{Conclusão}

Todos os doentes com doenças neuromusculares considerados com risco elevado de infeção grave no contexto de COVID-19 devem praticar distanciamento social estrito, preferencialmente confinamento ao domicílio e apenas quando estritamente necessário deslocações a locais públicos (farmácia, supermercado, hospital) devendo manter a distância de outras pessoas de pelo menos I metro, utilizar máscara cirúrgica e higienizar frequentemente as mãos (lavagem ou solução alcoólica). Os cuidados médicos devem ser sempre que possível, através de contacto telefónico com o seu médico assistente, se clinicamente apropriado. ${ }^{8}$

\section{Responsabilidades Éticas}

Conflitos de Interesse: Os autores declaram não possuir conflitos de interesse.

Suporte Financeiro: O presente trabalho não foi suportado por nenhum subsídio ou bolsa.

Proveniência e Revisão por Pares: Não comissionado; revisão externa por pares.

\section{Ethical Disclosures}

Conflicts of Interest: The authors have no conflicts of interest to declare.
Financial Support: This work has not received any contribution grant or scholarship.

Provenance and Peer Review: Not commissioned; externally peer reviewed.

\section{Referências}

1. World Health Organization. Coronavirus disease 2019 (COVID-19) situation report - 69. [Accessed March 29, 2020]. Available from: https://www.who.int/docs/default-source/ coronaviruse/situation-reports/20200329-sitrep-69-covid-19. pdf?sfvrsn $=8 \mathrm{~d} 6620 \mathrm{fa} 4$

2. Zheng Z, Peng F, Xu B, Zhao J, Liu H, Peng J et al. Risk factors of critical \& mortal COVID-19 cases: a systematic literature review and meta-analysis. J Infect. 2020 (in press).doi: 10.1016/j.jinf.2020.04.021

3. Centers for Disease Control and Prevention (CDC). People who are at higher risk for severe illness. [Accessed April 27, 2020]. Available from: https://www.cdc.gov/ coronavirus/2019-ncov/need-extra-precautions/people-at-higher-risk.html

4. Guidon A, Amato A. COVID-19 and neuromuscular disorders. Neurology. 2020 (in press). doi:10.1212/ WNL.0000000000009566

5. Guidance for the management of Myasthenia Gravis (MG) and Lambert-Eaton Myasthenic Syndrome (LEMS) during the COVID19 pandemic 23 March 2020 International MG/COVID Working Group. J Neurol Sci. 2020 (in press). doi: 10.1016/j. jns.2020.116803.

6. Veerapandiyan A, Connolly AM, Finkel RS, Arya K, Mathews $K D$, Smith EC et al. Spinal muscular atrophy care in the COVID-19 pandemic era. Muscle Nerve 2020 (in press). doi: $10.1002 /$ mus. 26903

7. Rajabally YA, Goedee HS, Attarian S, Hartung HP. Management challenges for chronic dysimmune neuropathies during the COVID-19 pandemic. Muscle Nerve. 2020 (in press). doi: $10.1002 /$ mus.26896.

8. Direção Geral da Saúde. Norma DGS n 007/2020 de 29/03/2020. Lisboa: DGS; 2020 